\title{
Biomecánica del complejo periastragalino
}

\author{
ANGULO CARRERE, M. ${ }^{\text {a }}$ T* Y LLANOS ALCÁZAR, L.F.** \\ Departamento de Enfermería II. Universidad Complutense de Madrid* \\ Departamento de Ciencias Morfológicas Universidad Complutense de Madrid**
}

\section{RESUMEN:}

Bajo el concepto funcional de «COMPLEJO PERIASTRAGALINO», se realiza una revisión de las característifas biomecánicas de las articulaciones que lo constituye.

Se estudia la movilidad de la articulación del tobillo, haciendo referencia a su papel en la transmisión de cargas y a su importancia en el mantenimiento de la estabilidad y congruencia articular de la unión del pie al extremo distal de la pierna, a traves de la existencia de un potente sistema ligamentoso.

Se revisan los conceptos de movilidad articular «triplanar», que caracteriza a las articulaciones del retro y mesopie, los movimientos «preferenciales» de cada una de estas uniones y, su implicación en los movimientos conjugados del «COMPLEJO ARTICULAR PERIASTRAGALINO».

Palabras clave: Articulación Tibioperoneoastragalina (TPA). Articulación Subastragalina (SA). Articulación Mediotarsiana (MT).

\section{SUMMARY}

Review of «Peritalar Joint» Biomechanics.

Study of ankle joint movility and it responsability in forces transfer, manteining the stability of talocrural union.

Review of «triplanar» movility concept and it caracteristic in rearfoot and midfoot joints. Explanation of "preference» movements of theses joints an its responsability of combine movements of the foot.

Key words: Tibiotalar Joint (TPA). Subtalar Joint (SA). Midtarsal Joints (MT).

\section{INTRODUCCIÓN:}

Los movimientos que realiza el pie en su conjunto, se corresponden con movimientos combinados que tienen lugar en los tres planos de referencia espacial: Rotación Interna-Externa (Plano Frontal), Flexión Dorsal y
Plantar (Plano Sagital) y Abducción-Adducción (Plano Transverso).

Durante la actividad normal, estos movimientos que tienen lugar entre la pierna y el pie, son combinaciones de desplazamientos articulares que son definidos como: INVERSIÓN (asociación de Flexión Plantar, Adducción y Rotación Externa del pie) y EVERSION (movimiento de Flexión Dorsal, Abducción y Rotación Interna del pie). ${ }^{1}$

La responsabilidad de cada una de las articulaciones implicadas en estos movimientos, depende de si se realizan bajo carga (cadena cinética cerrada) o sin apoyo del pie en el suelo (cadena cinética abierta).

Dadas estas características, el estudio biomecánico de la unión tibioperoneoastragalina y la de las articulaciones que conforman el retro y mesopie excede de la simple compresión de la anatomía y fisiología, debiendo encuadrarse en una superestructura funcional más amplia: el «Complajo Articular Periastragalino». ${ }^{2}$

Cuando se estudia la cinemática del pie, se observa que solo las articulaciones Metatarsofalángicas, las uniones del tarso con $2^{\circ}, 3^{\circ}$ y $4^{\circ}$ metatarsianos y las articulaciones Interfalángicas de los dedos, realizan movimientos aislados puros sobre los planos anatómicos de referencia que no afectan o implican el resto de las articulaciones del pie. ${ }^{3}$

Por el contrario, las articulaciones del tobillo, subastrastragalina, mediotarsiana y la unión del tarso con los metatarsianos $1^{\circ}$ y $5^{\circ}$, poseen ejes de movimiento cuya orientación es oblícua con respecto a los tres planos anatómicos de referencia. Esta orientación de sus ejes provoca que al moverse estas articualciones se origine un desplazamiento de los segmentos articulares, de forma simultánea, en los tres planos del espacio ${ }^{4}$.

Cuando, como en el caso referido, el eje de movimiento de una articulación está orientado de tal forma que constituye diferentes ángulo con los planos anatómicos de referencia, el movimiento originado se denomina «TRIPLANAR», lo que significa que, cada vez que se ejecuta el movimiento, este se traduce en desplazamiento sobre cada uno de los planos del espacio (fig. 1). En estos casos, cualquier intento de aislar los movimientos de la articulación en un sólo plano provocará una subluxación o un bloqueo de la articulación. ${ }^{3}$ 


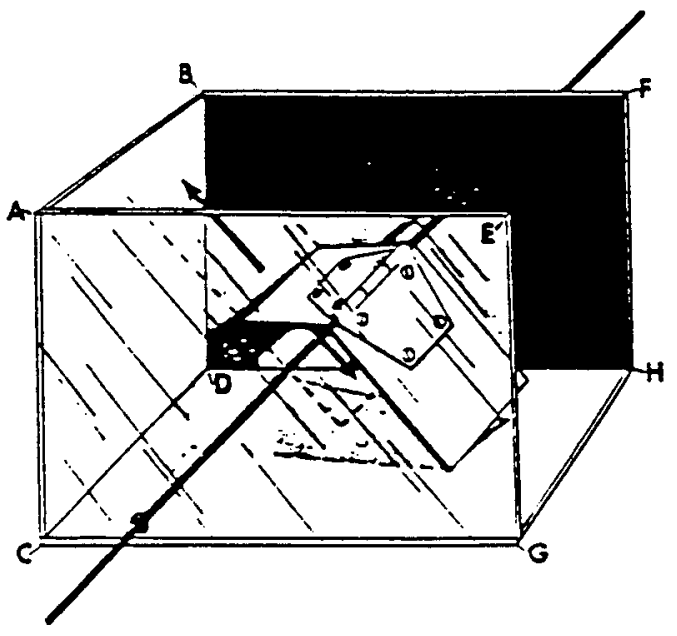

Figura 1. El movimiento que ejecuta la bisagra alrededor de su eje origina desplazamiento del segmento móvil en los tres planos del espacio (MOVIMIENTO TRIPLANAR). (Root ML, Orien WP, Weed JH; 1977.)

\section{ARTICULACIÓN DEL TOBILLO}

Esta articulación está establecida por la unión de la mortaja tibioperonea y la polea astragalina. Las extremidades distales de la tibia y peroné, se encuentran unidos a través de una potente sindesmosis anterior y posterior. Este sistema ligamentoso es el encargado de controlar la estabilidad de la pinza maleolar durante los desplazamientos que tienen lugar al realizarse los movimientos de pie, permitiendo la correcta transferencia de cargas en la articulación del tobillo ${ }^{5}$ que se prolonga con los sitemas trabeculares que atraviesan el esqueleto óseo del pie (sustentáculum tali, sistema talámico anterior y posterior y sistema aquíleocalcáneoplantar) ${ }^{6}$.

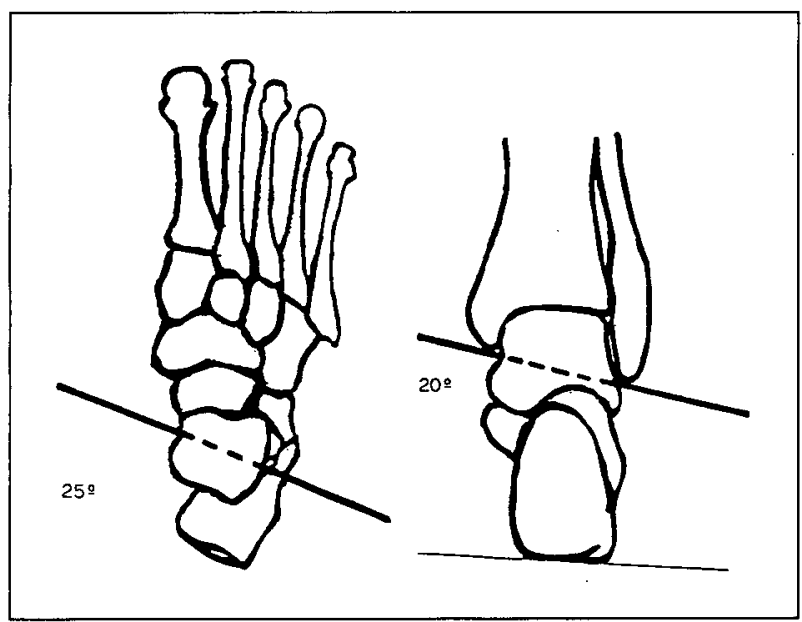

Figura 2. Orientación del eje de movimiento de la articulación del tobillo.
La articulación del tobillo posee un eje de movimiento oblícuo en relación a los planos del espacio, cuya inclinación, en el plano transverso, va a depender de la forma y longitud de ambos maleolos, pero que de forma general constituye un ángulo de aproximadamente 20 grados (fig. 2).

Este eje de movimiento del tobillo, permite realizar flexión dorso-plantar del pie y, además, debido a la inclinación que presenta, origina un desplazamiento asociado del astrágalo en el plano horizontal. Este desplazamiento se traduce en un movimiento de adducción en el transcurso de la flexión plantar y, por el contrario, de abducción durante la dorsiflexión. Estos movimientos asociados de la articulación, producidos en dos planos del espacio (sagital y horizontal); llevan a considerar al tobillo como articulación de tipo «helicoidal»?

Mecanicamente, la unión tibioperoneoastragalina, se ve sometida a solicitaciones en tracción en el transcurso de la flexión plantar $y$, por el contrario, a fuerzas de comprensión durante la flexión dorsal. ${ }^{8,9}$ La transmisión correcta de estas cargas así como el mantenimiento de la estabilidad y congruencia articualr, tal y como se ha referido anteriormente, es responsabilidad de los ligamentos tibioperoneo anterior y posterior.

Las fuerzas de comprensión que se ejercen sobre el tobillo al realizar la flexión dorsal del pie, unidas a la existencia de una asimetría en la polea astragalina (más ancha en su porción anterior) originan un desplazamiento obligado del peroné cuyo maleolo, de forma simultánea, realiza Abducción, Rotación Interna y Ascenso. Este movimiento del peroné se invierte durante la flexión plantar del pie (fig. 3).

\section{ARTICULACIÓN SUBASTRAGALINA}

Esta articulación está constituida por tres uniones anatómicas entre la porción inferior del astrágalo y la superior del calcáneo que se mueven simultaneamente alrededor de un mismo eje. Este eje denominado «eje de

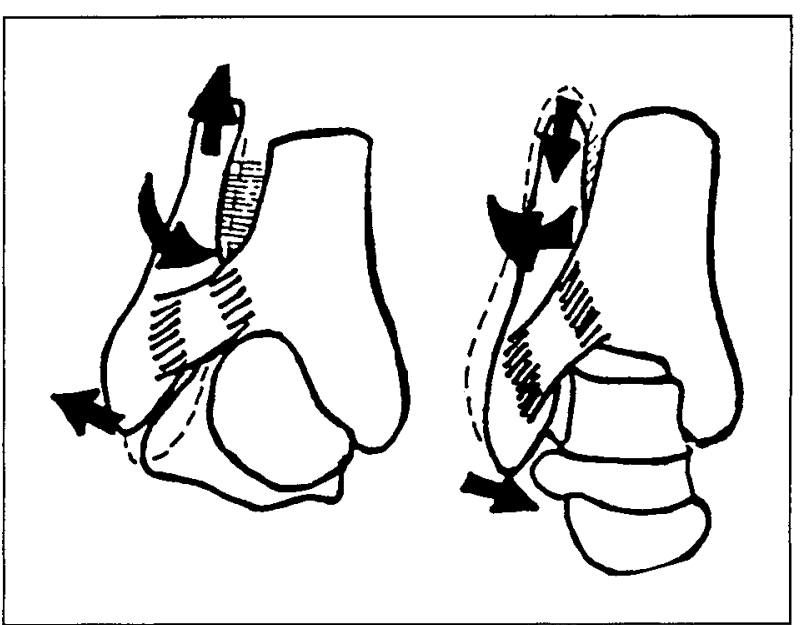

Figura 3. Desplazamientos del maleolo peroneo durante la Flexión Dorsal (A) y Flexión Plantar (B). 
Henke», sigue una dirección oblícua -forma ángulo de $42^{\circ}$ con el plano transverso y de $16^{\circ}$ con el plano sagital (fig. 4)- y origina, al moverse la articulación subastragalina, un desplazamiento conjunto en los tres planos del espacio. ${ }^{10}$

La unión astragalocalcánea interviene, como ya se ha descrito, en los movimientos combinados de INVERSIÓN- EVERSIÓN del pie, pero la implicación del calcáneo y astrágalo en ellos va a ser diferente dependiendo de si se trata de un movimiento realizado con el pie en carga (cadena cinética cerrada) o sin apoyo del pie en el suelo (cadena cinética abierta).

En este último caso, cadena abierta, el responsable del movimiento es el calcáneo, cuyo desplazamiento arrastra consigo a todo el pie, realizando ademas un movimiento de «listesis».11

Cuando la articulación subastragalina se halla sometida a carga (cadena cerrada), situación que tiene lugar durante la marcha, el astrágalo va a ser responsable de la mayor parte del movimiento que se produce en los planos sagital y transverso, mientras el calcáneo realiza los movimientos en el plano frontal. En el transcurso de la deambulación normal, tras el apoyo de talón, y debido a la resistencia que el suelo va a oponer a los desplazamientos del calcáneo, este va a quedar bolqueado para realizar cualquier tipo de movimeinto que no sea supinanción o pronación. De esta forma, va a ser el astrágalo quien origine el movimiento de Dorsiflexión y Ảbducción, con respecto al calcáneo, en el transcurso de la INVERSIÓN y, por el contrario, es responsable del desplazamiento en Flexión Plantar y Addución durante la EVERSIÓN (fig. 5).

La articulación subastragalina y la articulación del tobillo se comportan funcionalmente como un «CARDAN HETEROCINÉTICO» ya que sus ejes de movimiento están situados en planos diferentes..$^{12}$ Debido a esto, se van a producir movimientos a lo largo de unas direcciones preferenciales. Así por ejemplo, en la articulación Tibioastragalina se producirán movimientos de Flexión Dorsoplantar fundamentalmente $\mathrm{y}$, en menor proporción, de rotación o Abducción-Adducción; movimiento que, por el contrario, ocurrirán principalmente en la articualción subastragalina (tabla 1).
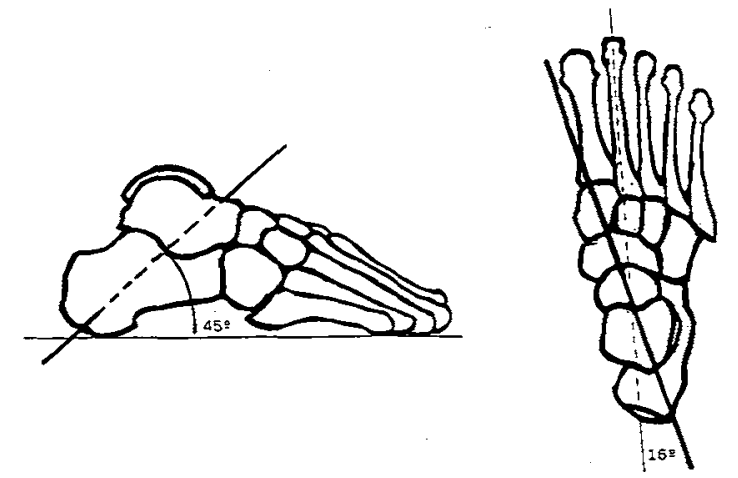

Figura 4. Orientación del eje de mvimiento de la articulación Subastragalina.
TABLA 1

ESQUEMA DE MOVIMIENTOS «PREFERENCIALES" DEL COMPLEJO ARTICULAR PERIASTRAGALINO

\begin{tabular}{|c|c|c|}
\hline $\begin{array}{c}\text { Flexión plantar } \\
+ \\
\text { Flexión dorsal }\end{array}$ & $\begin{array}{l}\text { Inversión } \\
\text { Eversión } \\
\text { Listesis }\end{array}$ & $\begin{array}{l}\text { (Rotación) } \\
\text { Pronación } \\
\text { Supinación }\end{array}$ \\
\hline $\begin{array}{c}\text { (Articulación } \\
\text { tibio-astragalina }\end{array}$ & $\begin{array}{l}\text { (Articulación } \\
\text { subastragalina }\end{array}$ & $\begin{array}{l}\text { (Articulación } \\
\text { mediotarsiana }\end{array}$ \\
\hline
\end{tabular}

Scott y cols. ${ }^{13}$, han observado recientemente cómo los movimientos que tiene lugar en estas articulaciones son opuestos. Mientras en la Tibiotarsiana se produce una inversión durante la fase de apoyo de talón, en la Subastragalina se origina un movimiento de eversión. Estos movimientos se van a invertir en el transcurso del apoyo completo del pie durante la marcha y tienen como misión absorber parte de la rotación, que sobre el plano transverso, realiza la tibia durante la deambulación, además de amortiguar la carga y favorecer el efecto «mecedora» del pie mientras tiene lugar la fase de apoyo monopodal.

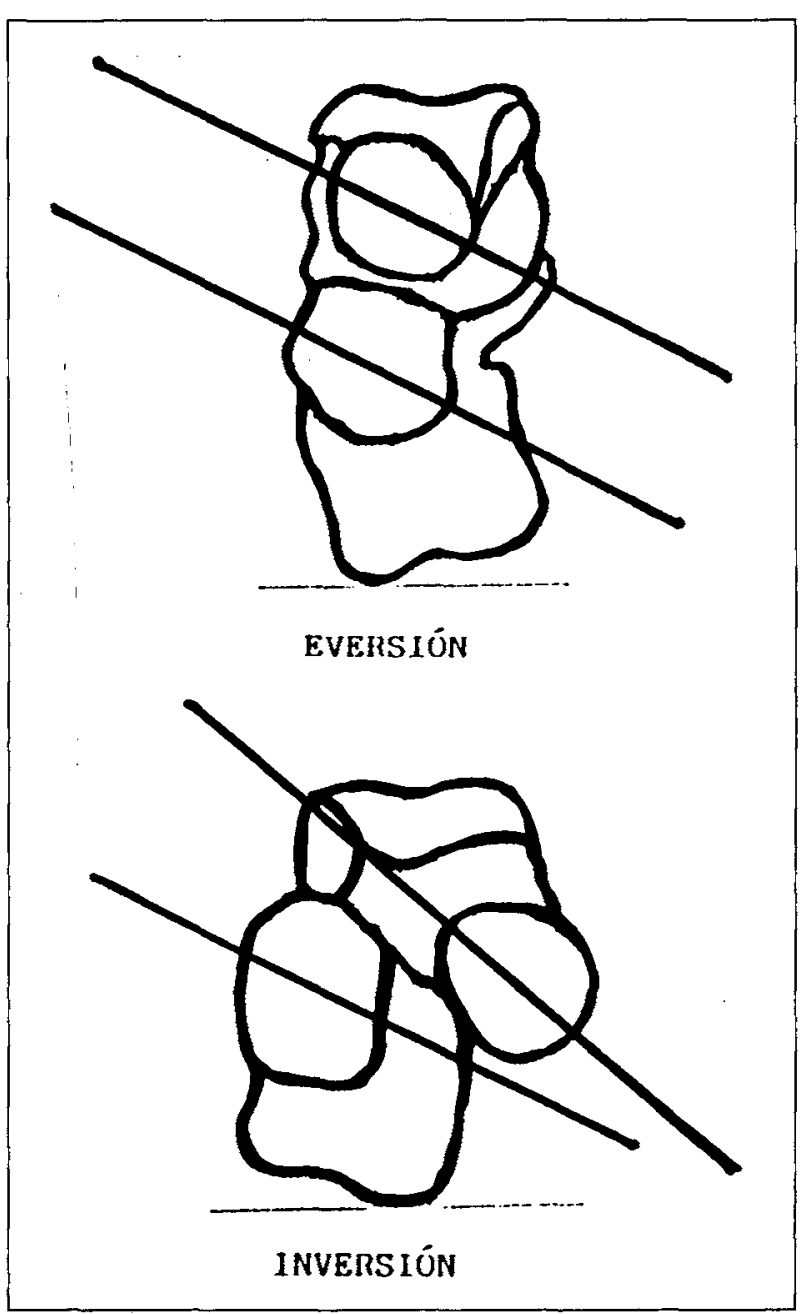

Figura 5. Movimientos de la articulación Subastragalina en cadena cerrada y orientación de los ejes de la articulación Mediotarsiana. 
Para que se efectúe correctamente la transmisión hacia el pie del movimeinto de rotación que tiene lugar en la tibia, es imprescindible una buena orientaicón del eje de Henke. En condiciones normales, como ya se ha referido, este eje de movimiento de la articulación subastragalina, forma un ángulo de $45^{\circ}$ con el plano transverso; esta inclinación origina que, por cada grado de rotación de la tibia sobre el plano horizontal, se produzca un grado de rotación de la articulación subastragalina sobre el plano frontal. De esta forma, durante la fase de apoyo de la marcha, cuando el astrágalo se abduce por la rotación interna de la tibia, el calcáneo realiza un movimiento de pronación y, al contrario, cuando se produce rotación externa de la tibia, el astrágalo se abduce y se origina una supinación del calcáneo. Estos movimientos sólo tiene lugar con el pie sometido a carga (cadena cerrada) ya que, cuando no está apoyada, la rotación de la pierna producirá cambios en la orientación del pie, pero no movimientos. 14

En los casos en los que el eje de Henke se oriente más paralelo al plano transverso, existirá mayor movilidad del calcáneo (supinación o pronación) por cada grado de rotación de la tibia. Cuando el eje se verticaliza y sobrepasa los $45^{\circ}$ con respecto al plano horizontal, se observa mayor rotación tibial que movimiento del calcáneo. ${ }^{3}$

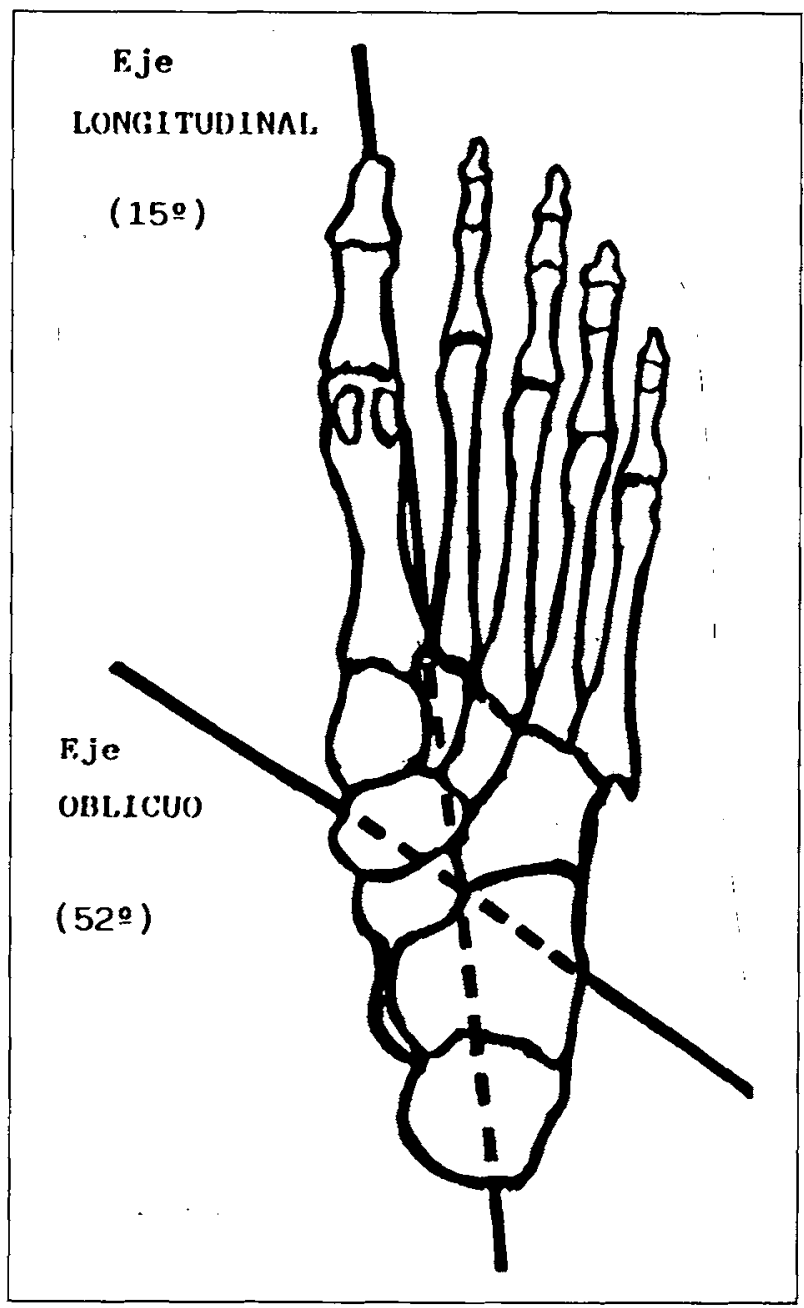

Figura 6. Esquema en el que se muestra la orientación de los ejes de la articulación Mediotarsiana.

\section{ARTICULACIÓN MEDIOTARSIANA}

Está constituída por la unión de dos articulaciones que funcionan conjuntamente alrededor de dos ejes comunes de movimiento. Estos ejes son oblícuos con diferente orientación en el pie. El eje LONGITUDINAL forma un ángulo con el plano transverso de $15^{\circ}$ y de $9^{\circ}$ con el plano sagital. El eje OBLÍCUO, origina un ángulo de 52 grados con el plano transverso y de 57 con el sagital (fig.6).

Sobre el primero de los ejes se van a producir movimientos de inversión-eversión fundamentalmente $y$, alrededor del eje oblícuo van a tener lugar los movimientos de abducción-adducción y flexión dorsal y plantar. ${ }^{14}$

La ventaja de la existencia de estos dos ejes en la articulación mediotarsiana, es que va a permitir dejar libre el antepié para producir el movimiento de inversióneversión sobre el plano frontal sin originar ningún movimiento sobre los otros ejes del espacio, lo que ayuda a compensar la inversión-eversión que pueda tener lugar en el retropié. ${ }^{9}$

Cuando existe una excesiva verticalización del eje Oblícuo de la articualción mediotarsiana, se origina un aumento del movimiento de Abducción-Adducción con disminución de la Flexión Dorsoplantar. Esto es debido al excesivo movimiento de Abducción del antepié, asociado a Flexión Dorsal, que tiene lugar durante la fase de apoyo unipodal de la marcha; el retropié aumenta su adducción, originando un anormal desplazamiento de la componente vertical del peso del cuerpo, lo que da lugar a una excesiva pronación de la articulación subastragalina. ${ }^{3}$

Como resumen, podríamos concluir que el complejo funcional «PERIASTRAGALINO» va a estar constituído por uniones articulares cuyos ejes de movimeinto son oblícuos en relación con los tres planos espaciales de referencia, originando movimientos «TRIPLANARES» que van a permitir una mejor distribución y amortiguación de las diferentes fuerzas a las que se ve sometido el pie durante la marcha.

\section{BIBLIOGRAFIA}

1. KAPANDJI I.A.. Cuadernos de Fisiología Articular: Miembro Inferior. $3^{\text {a }}$ Ed. Masson S.A... Barcelona, 1984.

2. LLANOS ALCAZAR L.F.. Patamecánica del Pie. Bol. Soc. Esp. Hidrol Med. Vol VIl1: 13-18.

3. ROOT M.L., ORIEN W.P., WEED J.H. Normal and Abnormal Function of the Foot. Clinical Biomechanics Corporation. Los Angeles. 1977

4. INMAN V. The Joints of the Ankle. Williams and Wilkins. New York. 1976.

5. BLAIMONT P., LIBOTTE M., KLEIN P. Biomécanique de la Tibio-tarsiénne. Implications Cliniques. Cahiers d'enseignement de la SOFCOT. 1986: $21-36$.

6. VILLADOT PERICÉ A. Quince Lecciones sobre Patología del Pie. Editorial Toray S.A.. Barceiona. 1989.

7. MURPHY N., ALLARD P., AISSAUI R.. Helical Motion of the Ankle. Proceedings of NACOB II. Chicago, August 24th - 18th, 1992.

8. PROUBASTA I. Estudio Cinemático del Tobillo. Avances Traum 1992; 221:5 - 11 .

9. SAMMARCO G.J., BURSTEIN A.M., FRANKEL V.H.. Biomechanics of the Ankle: A Kinematic Study. Orthop Clin North Am 1973; 41:75-76.

10. DONATELLI R:. The Biomechanics of the Foot and Ankle. F.A. Davis Company. Philadelphia, 1990.

11. MANN R.A.: Biomechanics, en «Disorders of the Foot» Vol. 1 1982; $37-67$. W!B! Saunders company.

12. SCOTT S.H., WINTER D.A.. Talocrural and Talocalcaneal Joint Kinematics and Kinetics During Stance Phase of Walking. J. Biomech 1991; 248:743-752.

13. NUNEZ-SAMPER M., LLANOS ALCAZAR L.F., Biomecănica, Međicina y Cirugía del pie. Copynorte S.L.. Madrid. 1989.

14. GERSHMAN S.A. Literatura Review of Midtarsal Joint Motion. Clin Podiatr Med Surg 1988; 52: 385-391. 